\begin{tabular}{|c|c|}
\hline Title & Effect of alkanethiol molecular structure on sensitivity of surface plasmon resonance sensor \\
\hline Author(s) & Suherman; Morita, Kinichi; Kawaguchi, Toshikazu \\
\hline Citation & $\begin{array}{l}\text { Sensors and A ctuators B: Chemical, 210, } 768.775 \\
\text { https:/doi.org/10.1016/.snb.2015.01.026 }\end{array}$ \\
\hline Issue Date & 2015-04 \\
\hline DOC URL & http:/hdl.handle.net/2115/65195 \\
\hline Rights & $\begin{array}{l}\text { (2) 2015. Inis manuscript version is made avallable under the CC-BY-IC-IVD } 4.0 \text { IIcense } \\
\text { http://reativecommons.org/icenses/by-nc-nd/4.0/ }\end{array}$ \\
\hline Rights(URL) & http:/creativecommons.org/icenses/by-nc-nd/4.0/ \\
\hline Type & article (author version) \\
\hline File Information & Sensors and A ctuators B 210 (2015) 768 775.pdf \\
\hline
\end{tabular}

Instructions for use 


\section{Effect of Alkanethiol Molecular Structure on Sensitivity of Surface Plasmon Resonance Sensor}

Suherman $^{1,2}$, Kinichi Morita ${ }^{3}$, Toshikazu Kawaguchi ${ }^{* 1}$

${ }^{1}$ Division of Environmental Materials Science, Graduate School of Environmental Science and Section of Materials Science, Faculty of Environmental Earth Science, Hokkaido University, Sapporo 060-0810, Japan.

${ }^{2}$ Department of Chemistry, Faculty of Mathematics and Natural Sciences, Universitas Gadjah Mada, Sekip Utara Kotak Pos 21 BLS Yogyakarta 55281, Indonesia

${ }^{3}$ New Business Development Office, Ushio Inc. 6-1 Ohtemachi 2-chome, Chiyoda-ku, Tokyo 100-8150, Japan.

*Corresponding Author

Email: t_kawa@ees.hokudai.ac.jp

Tel.: +81-11-706-2289

Fax: +81-11-706-2289 


\section{Abstract}

This work aims to find the key factor determining the detection limit for an indirect competitive inhibition immunoassay using surface plasmon resonance (SPR) sensing. In our previous work, the thiol solution concentration used in a self-assembly process highly affected the alkanethiol monolayer structure on the sensor surface [Suherman et al., 2014]. It was noticed that the monolayer structure determined the immunoassay sensitivity due to the orientation and the surface concentration of antigen in domain structure of monolayer. To study the effect of orientation of antigen, we examined here three types of alkanethiol compounds: dithiobis(succinimidyl

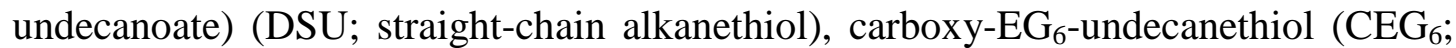
flexible-chain alkanethiol), and 3,3'-dithiobis[ $N$-(5-amino-5carboxypentyl)propionamide- $N, N^{\prime}$-diacetic $\quad$ acid $] \quad\left(\mathrm{C}_{2}\right.$-NTA; $\quad$ three-branched alkanethiol). By electrochemical reductive desorption, the surface concentration of DSU, $\mathrm{CEG}_{6}$, and $\mathrm{C}_{2}$-NTA were estimated to be $6.6 \times 10^{-10}, 8.5 \times 10^{-10}, 4.6 \times 10^{-10}$ mol cm${ }^{-2}$, respectively. Subsequently, SPR suggested that the ratios of immobilized antigen (clenbuterol) per thiol were $0.13,0.16$, and 0.15 for $\mathrm{DSU}, \mathrm{CEG}_{6}$, and $\mathrm{C}_{2}-\mathrm{NTA}$, respectively. This suggests that the amount of immobilized clenbuterol was defined by the molecular size of clenbuterol. Sensor surface structures on a molecular scale were evaluated using high-resolution scanning tunneling microscopy (STM), which showed tilted clenbuterol with $\theta_{D S U}>\theta_{C E G 6}>\theta_{C 2-N T A}$. For SPR sensing of clenbuterol, $\mathrm{C}_{2}$-NTA showed the highest sensitivity (LOD $=10 \mathrm{ppt}$ ) among the examined alkanethiols, because clenbuterol formed a laid-on structure. Based on kinetics, the key factor for sensing performance is discussed. 
Keywords: alkanethiol monolayer, surface plasmon resonance, clenbuterol, indirect competitive inhibition immunoassay.

\section{Introduction}

Biosensors are analytical devices that measure binding of analytes to surfaces, a binding biomolecules (e.g., DNA, protein, enzyme, and antibody) induce a signal in a physichochemical transducer [1-4]. Several developments in biosensors have been reported, such as fluidic design [5-7], sensor surface immobilization [8-11], advances in detection methods [12-15], and data analysis [15-16]. An ideal biosensor is reliable, selective, sensitive, quick to respond, and inexpensive to fabricate. However, to achieve a robust biosensor, accurate design and control of the interfacial chemical reaction are important in biosensing and surface engineering [17]. Among the interfaces with molecular recognition, self-assembled monolayers (SAMs) have received attention because of advantages such as excellent stability and direct integration with biomolecular materials, and the possibility of molecular-level control in applications such as sensors, catalysts [18-20]. SAMs can be easily prepared on metal surfaces, because adsorbates (e.g., thiol and silane) can spontaneously organize into crystalline or semicrystalline structures. Thus, these adsorbates form highly ordered and highly oriented monolayers on surfaces. Therefore, in this study, we investigated the control of the orientation of an end group of an adsorbate in order to find the key factors that determine sensitivity of an immunoassay. We propose that the sensor surface structure plays an important role in interactions with the target compound.

In our previous work [21], we fabricated a biosensor chip using succinimidylterminated propanethiol prepared from dithiobis(succinimidyl propionate) (DSP). We 
found that the surface morphology of the SAM depended on DSP solution concentration used in self-assembly process. Moreover, domains of antigen (clenbuterol; target molecule) and blocking agent (ethanolamine) were strongly correlated with the DSP solution concentration. We concluded that the domain structure highly affects the immunoassay sensitivity.

Some attempts for the orientation control by using mixed alkanethiol monolayer were reported [22-24]. Mixed monolayers were prepared from mixed solution of long- and short-alkyl chain alkanethiols. However, they often formed the phaseseparated domain, which consisted of the single component domain. Thus, we examined three types of alkanethiol to explore the orientation control in this work; as illustrated in Fig. 1: (1) straight-chain alkanethiol prepared from dithiobis(succinimidyl undecanoate) (DSU), (2) flexible-chain alkanethiol prepared

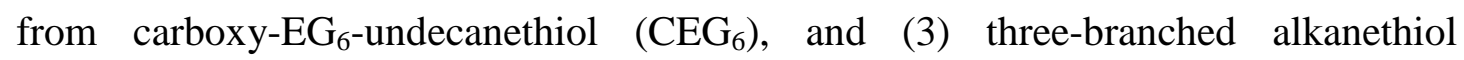
(dendrimer alkanethiol, $\mathrm{C}_{2}$-NTA) prepared from 3,3'-dithiobis[ $N$-(5-amino-5carboxypentyl)propionamide- $N, N^{\prime}$-diacetic acid]. DSU is a well-known succinimidylterminated alkanethiol for biosensors [25-27]. DSU has a long straight alkyl chain and a succinimidyl group in the head group; it forms a highly oriented and concentrated monolayer on Au surfaces [27]. $\mathrm{CEG}_{6}$ is widely used as a biochemical linker because of its ability to prevent nonspecific adsorption [28-31]. $\mathrm{C}_{2}$-NTA is of interest to researchers because of its well-defined molecular structure and interesting properties, such as low intrinsic viscosity and solubility higher than analogues linear molecules [32]. Thus, we surmised that these three alkanethiols could covalently immobilize the target analyte in different orientations on the sensor surface.

Our target analyte was clenbuterol, which is a controversial substance in sports because of doping. Clenbuterol is a member of the $\beta$-adrenergic agonist family, 
and its primary medical use is as a bronchodilator agent in asthma treatments [33-34]. Farmers illegally use this compound to boost muscle tissue production of their livestock, such as pigs, cows, and turkeys to increase profit. Because doping is a serious issue in global competitions, such as the soccer World Cup, the Olympics, and Tour de France, attention to this $\beta$-adrenergic agonist compound has intensified because of its easy uptake and deposition in the human body with daily meat consumption. Thus, developing a reliable detection method with high sensitivity and rapid detection time is necessary.

In this study, surface plasmon resonance (SPR) was used to sense clenbuterol because it satisfies the above requirements for practical use. SPR is well known as a highly mass transducer based on surface plasmon phenomena. SPR monitors the dielectric constant change at the interface caused by a binding event of target analyte and bio-interfacial material. Even though the limit of detection (LOD) for measuring mass change using our SPR sensor is approximately $30 \mathrm{pg} / \mathrm{cm}^{2}$, the detection of a small-sized target in a short time remains difficult. Therefore, we used an indirect competitive inhibition immunoassay [14]. Compared to other immunoassays, this immunoassay has a better sensitivity for detection of a small analyte, because the mass transducer react to mass change due to binding of an antibody instead of an antigen (a lightweight analyte). However, the mechanism for this enhancement remains unclear. For rapid detection, the sensor monitors the initial process of immunoreaction, which is affected by the concentration of analyte according to the Langmuir equation. Therefore, we also discuss the effect of kinetics on the immunoassay sensitivity.

\section{Experimental}




\subsection{Materials}

Potassium hydroxide, ethanol, and chloroform were obtained from WAKO, Japan, and sodium hydroxide was obtained from Junsei Chemical Co., Ltd., Japan. DSU, $\quad$ CEG $_{6}, \quad \mathrm{C}_{2}$-NTA, $\quad N$-(3-dimethylaminopropyl)- $N$--ethylcarbodiimide hydrochloride (EDC) and phosphate-buffered saline (PBS) were purchased from Sigma Aldrich, USA, and $N$-hydroxysuccinimide (NHS) was purchased from FLUKA, Germany. Methanol was obtained from Dojindo, Japan. Clenbuterol hydrochloride (CLB) and anti-clenbuterol mouse monoclonal IgG antibody (Ab) were ordered from LKT Laboratories, Inc., USA, and Novus Biologicals, USA, respectively. Refractive index matching fluid (refractive index $=1.518$ ) was obtained from Cargille Labs, USA. All chemicals were of reagent or higher grade, and Millipore Milli-Q water (18.2 $\mathrm{M} \Omega \mathrm{cm}$ ) was used throughout the experiment.

\subsection{Preparation of Au substrates}

Au chips were prepared by sputtering deposition of an Au plate onto glass substrates (BK7 type, $20 \mathrm{~mm} \times 13 \mathrm{~mm} \times 0.7 \mathrm{~mm}$ from Matsunami Glass Ind., Ltd., Japan) using a JFC-1600 Fine coater from JEOL, Japan after sufficient sonication in soapy water (10\% Contrad 70 detergent; Fisher scientific, USA) followed by rinsing with plenty of Millipore water.

Prior to gold sputter deposition, the glass substrates were cleaned by plasma discharge at $15-\mathrm{W}$ under $2.0-2.5 \mathrm{~Pa}$ in air. $50 \mathrm{~nm}$ of $\mathrm{Au}$ was sputtered onto the glass chips under 2.0-2.5 Pa. Au chips prepared by this method were used for all measurements.

Surface densities on text were corrected with the surface roughness of Au-chip $(\delta=1.50)$, which was calculated from the reduction charge density of Au-O using cyclic voltammetry in $0.1 \mathrm{M}$ sulfuric acid. 


\subsection{Electrochemistry}

The electrochemical measurements were conducted in an electrochemical cell holding using an HZ-5000 Automatic Polarization System from Hokuto Denko, Japan. All experiments were conducted using a conventional three-electrode system with the Au chip as the working electrode, a platinum wire as the counter electrode, and an $\mathrm{Ag} / \mathrm{AgCl}$ (saturated $\mathrm{KCl}$ ) as the reference electrode. A $0.1 \mathrm{M} \mathrm{KOH}$ aqueous solution was used as the electrolyte and it was deaerated with $5 \mathrm{~N}$ Ar gas in a glove box for approximately 30 min before each experiment.

\subsection{Surface morphology using scanning tunneling microscopy (STM)}

The surface morphology was investigated using a Nanoscope STM III (Digital Instruments, USA). For imaging, Pt/Ir (80/20) mechanically cut tips (diameter $=0.25$ mm, Bruker, USA) were used with a current of a few hundred pA and a positive bias voltage typically between 0.5 and $1 \mathrm{~V}$. The instrument was equipped with a lowcurrent converter MMS-STMLC (Machine Monitoring System-Single Topology Maximum Lifetime Convergecast).

\subsection{SPR sensing}

The SPR experiments were performed using an SPR-670 apparatus (Nippon Laser Electronics, Japan) equipped with a fully automated flow system consisting of a plunger pump and an injector. The Au chip was attached to a semi-cylindrical prism using the refractive index matching liquid. Red light $(670 \mathrm{~nm})$ emitted from a Ni-Cd laser reflected off the Au-coated glass plate at attenuated total reflectance (ATR) angles, and the reflected light intensity was recorded using a CCD camera. The reflectance angle at which the light intensity is minimum, called SPR angle, was recorded with respect to time. All experiments were conducted in an air-conditioned $\operatorname{room}\left(25^{\circ} \mathrm{C}\right)$. 


\subsection{Fabrication of the sensor surface}

Fig. 2 shows the SPR sensorgram of the fabrication process. First, the thiol monolayer was prepared by immersion and self-assembly with the flow rate of $5 \mu \mathrm{L}$ $\min ^{-1}$ from a $5 \mathrm{mM}$ thiol solution using DSU, $\mathrm{CEG}_{6}$, or $\mathrm{C}_{2}$-NTA. Since the DSU solution was diluted in chloroform, SAM fabrication using the DSU solution was performed outside of the SPR flow system to avoid damage to the PEEK tubes from the chloroform.

To convert from a carboxyl group to a succinimidyl group, EDC/NHS (0.2 M/0.05 M) was used. Subsequently, the clenbuterol solution (PBS solution containing $1 \mathrm{mg} \mathrm{mL}{ }^{-1}$ of clenbuterol) was injected for $40 \mathrm{~min}\left(5 \mu \mathrm{L} \mathrm{min}{ }^{-1}\right)$. In this process, the succinimidyl group was replaced by clenbuterol. Although sufficient clenbuterol flowed over the monolayer surface terminated with succinimidyl groups, not all of the succinimidyl groups reacted. To elinimate the unreacted succinimidyl groups, ethanolamine (PBS solution containing $1 \mathrm{mg} \mathrm{mL}^{-1}$ blocking agent) was also injected for $40 \mathrm{~min}$; therefore, unreacted succinimidyl groups were totally replaced by ethanolamine. Prepared sensor surfaces were used for sensing immediately after the fabrication process. These sensor surfaces should be very stable, because their fabrication involved only covalent bonds (S-Au, S-S, and NHCO) [35].

\subsection{Immunoassays}

For detecting clenbuterol, the indirect competitive inhibition immunoassay technique was employed [13, 35-37]. In this study, the antibody solution (PBS solution containing $1 \mu \mathrm{g} \mathrm{mL} \mathrm{m}^{-1}$ of $\mathrm{Ab}$ ) was premixed with a sample solution (PBS solution containing clenbuterol) before injection into the sensing system. As the mixed solution flowed over the sensor surface, the SPR sensed the dielectric constant change at the interface due to the binding of the unreacted $A b$ to clenbuterol 
immobilized on the sensor surface. After detection, Ab could be detached from the sensor surface by $0.1 \mathrm{M}$ sodium hydroxide and be ready for detection of clenbuterol again. Here, we confirmed reproducibility by using different sensor chips $(\mathrm{N}>3)$, and each plot was averaged within 10\% experimental error bars.

\section{Results and Discussion}

\subsection{Characterization of the sensor surfaces}

Electrochemical reductive desorption was performed to investigate the structure of the alkanethiol monolayers (Fig. 3). Since the S-Au bond can be electrochemically cleaved by a one-electron reaction; the surface concentration of the monolayer can be estimated from the charge density, which is calculated from the integration of the reduction current.

The reduction currents showed several peaks in the range of -0.9 to $-1.35 \mathrm{~V}$. This is characteristic of reductive desorption on a polycrystalline Au electrode [38]. By comparing the peak positions in the voltammograms to similar systems reported on previously, we assigned the peak at approximately $-0.90 \mathrm{~V}$ to desorption of alkanethiols from the terraces [39], and the peaks at -1.08 to $-1.37 \mathrm{~V}$ to the desorption of alkanethiols adsorbed on the steps of polycrystalline Au [40-41].

The surface concentrations of the DSU, $\mathrm{CEG}_{6}$, and $\mathrm{C}_{2}-\mathrm{NTA}$ (were calculated using equation mentioned in Supporting Information, and were already corrected by the roughness factor of 1.50 ) thiols were $6.6 \times 10^{-10}, 8.5 \times 10^{-10}$ and $4.6 \times 10^{-10} \mathrm{~mol}$ $\mathrm{cm}^{-2}$, respectively. If we further consider that the $-30 \%$ error due to the double layer charging current could be reflected in the surface concentrations calculated from the total reductive desorption charge, these concentrations are comparable to the result found from the angle shift of SPR and STM image calculations (with the assumption 
that each molecule of DSU, CEG , and $\mathrm{C}_{2}$-NTA occupied $0.3,0.27$, and $0.6 \mathrm{~nm}^{2}$, respectively; Fig. S1 in Supporting Information), as summarized in Table 1.

From the SPR angle shift, the ratios of immobilized antigen (clenbuterol) per thiol were estimated as $0.13,0.16$, and 0.15 for $\mathrm{DSU}_{2} \mathrm{CEG}_{6}$, and $\mathrm{C}_{2}-\mathrm{NTA}$, respectively. These ratios are very close. This suggests that only one carboxy group in the three branches of $\mathrm{C}_{2}$-NTA bonded to clenbuterol. These ratios are reasonable assuming clenbuterol $\left(0.67 \mathrm{~nm}^{2}\right)$ covered the close-packed alkanethiol monolayer.

In order to directly observe the sensor surface structure on a molecular scale, high-resolution STM was employed, as shown in Fig. 4. The DSU monolayer had an interesting stripped appearance, though the stripped pattern did not predominate on the surface. These striped features disappeared after clenbuterol and ethanolamine immobilization. The height of each stripe was $0.17 \mathrm{~nm}$, which is slightly lower than the diameter (i.e., $0.21 \mathrm{~nm}$ ) of a succinimidyl group (Fig. S2 in Supporting Information). Considering the size of the tilted succinimidyl group, the width of each stripe could consist of three to four succinimidyl groups per line. The lines were formed on top of an underlying gold surface. Thus, the lines were somewhat curved. On $\mathrm{CEG}_{6}$, atomically minor lines were observed. We think that these minor lines consisted of carboxyl groups forming hydrogen bonds with each other. After immobilization of clenbuterol and ethanolamine, blurred rings of clenbuterol were partially observed. In contrast, the molecular structure of clenbuterol was clearly seen on $\mathrm{C}_{2}$-NTA. In summary, the order of tilt angle of clenbuterol was as follows: $\theta_{\mathrm{DSU}}>$ $\theta_{\text {CEG6 }}>\theta_{\mathrm{C2}-\mathrm{NTA}}$. The precise tilt angles could not be determined because this was a polycrystalline Au surface. We also observed that the domain boundaries of clenbuterol and ethanolamine were not clear due to mixed distribution on the sensor surface (Fig. S3 in Supporting Information). 


\subsection{Detection of clenbuterol}

Figs. 5A-C show SPR sensorgram of immunoreactions with clenbuterol/ethanolamine immobilized DSU, CEG ${ }_{6}$, and $\mathrm{C}_{2}$-NTA sensor surfaces, respectively. While the Ab standard solution flows over the sensor surfaces for $250 \mathrm{~s}$ (from $t=100 \mathrm{~s}$ to $t=350 \mathrm{~s}$ ), the resonance angle gradually shifted higher with binding of $\mathrm{Ab}$ to the antigen immobilized on the sensor surface. The difference in the resonance angle before $(t=100 \mathrm{~s})$ and after $(t=400 \mathrm{~s})$ the immunoreaction is the total resonance angle shift $(\Delta \theta)$. This value was used for calculation of the surface concentration.

After the immunoreaction, the sensor surface is regenerated by a solution of 0.1 M sodium hydroxide till resonance angle reverted to the initial angle. Through such a regeneration process, the sensor surface was reused for over 100 immunoreactions. Each immunoreaction-regeneration cycle took only $1000 \mathrm{~s}$.

For detection of clenbuterol, an indirect competitive inhibition immunoassay was employed. Clenbuterol standard solution was premixed with Ab standard solution in advance. Then, $\Delta \theta$ was evaluated with respect to the clenbuterol solution concentration, as shown in Figs. 5a-c. As a result, typical sigmoid curves were obtained. The limits of detection (LOD) of clenbuterol were determined as $50 \mathrm{ppt}$ (DSU), 25 ppt $\left(\mathrm{CEG}_{6}\right)$, and 10 ppt $\left(\mathrm{C}_{2}\right.$-NTA) from 85\% of the maximum angle change.

The primary affinity constant $\left(K_{1}\right)$ of $\mathrm{Ab}$ to the clenbuterol-immobilized sensor surface and the maximum angle shift of the SPR after Ab injection $\left(\Delta \theta_{0, \max }\right)$ were determined using a Langmuir-type adsorption mode [42-46]. $K_{2}$, which is defined as the secondary affinity constant $K_{1}$ of $\mathrm{Ab}$ to the free clenbuterol in the 
solution, was also determined. These values were estimated from the slope of the Langmuir adsorption isotherm plot in Fig. 6.

For DSU, $\mathrm{CEG}_{6}$, and $\mathrm{C}_{2}$-NTA, respectively, $K_{1}$ was $5.2 \times 10^{8}, 9.7 \times 10^{8}$, and $4.8 \times 10^{8} \mathrm{M}^{-1}$; and $K_{2}$ was $(18.1 \pm 7.9) \times 10^{8} \mathrm{M}^{-1}$. Correlating these kinetic parameters for the various thiol sensor surfaces to the detection of clenbuterol, a lower $K_{1}$ in combination with a higher $K_{2}$ leads to better sensitivity (10 ppt). This result agrees with the finding of our previous study of clenbuterol detection using a DSP alkanethiol monolayer, in which a lower $K_{1}$ corresponded to the highest sensitivity [21]. Particularly, $K_{1}$ is determined by the distribution and molecular structure of antigen exposed to the liquid phase. Here, the amounts of antigen were almost the same for each thiol. Moreover, clenbuterol was well distributed on all of the surfaces, because separated domains of clenbuterol and ethanolamine were not observed in the STM images. This suggests that $K_{1}$ is strongly affected by the orientation of antigen, i.e., the ordering of tilt angle ( $\theta_{\mathrm{DSU}}>\theta_{\mathrm{CEGG}}>\theta_{\mathrm{C} 2-\mathrm{NTA}}$, as determined by STM) was the reverse of the ordering of sensitivity [50 ppt (DSU) $<25$ ppt $\left(\mathrm{CEG}_{6}\right)<10$ ppt $\left(\mathrm{C}_{2^{-}}\right.$ NTA)]. A higher tilt angle of antigen leads to a higher affinity constant but a lower sensitivity. In addition, this suggests that the anti-clenbuterol monoclonal antibody recognizes the tail group of clenbuterol in the molecular structure, because tail-up structure of clenbuterol showed high affinity to Ab. We believe this consideration will be helpful in future understanding of the recognition mechanism of Ab.

\section{Conclusions}

To find the key factor determining the detection limit of an indirect competitive inhibition immunoassay, the orientation effect of the antigen at the sensor surface was examined. We used three types of alkanethiol compounds; a straight-chain alkanethiol 
(DSU), a flexible-chain alkanethiol $\left(\mathrm{CEG}_{6}\right)$, and a three-branched alkanethiol $\left(\mathrm{C}_{2}{ }^{-}\right.$ NTA). Electrochemical reductive desorption revealed the surface consentration of for DSU, $\mathrm{CEG}_{6}$, and $\mathrm{C}_{2}$-NTA were $6.6 \times 10^{-10}, 8.5 \times 10^{-10}$, and $4.6 \times 10^{-10} \mathrm{~mol} \mathrm{~cm}^{-2}$, respectively. SPR showed that the amounts of immobilized antigen (clenbuterol) per thiol were approximately $0.13,0.16$ and 0.15 for $\mathrm{DSU}_{,} \mathrm{CEG}_{6}$, and $\mathrm{C}_{2}-\mathrm{NTA}$, respectively. Thus, they had almost the same amount of clenbuterol on the sensor surface. Molecular structures at the sensor surfaces were observed using highresolution STM. The order of the tilt angle of clenbuterol was $\theta_{\mathrm{DSU}}>\theta_{\mathrm{CEGG}}>\theta_{\mathrm{C2}-\mathrm{NTA}}$. Moreover, clenbuterol was well distributed on the entire surfaces, because separated domains of clenbuterol and ethanolamine were not observed in STM images. In SPR sensing of clenbuterol, $\mathrm{C}_{2}$-NTA showed the highest sensitivity (LOD = $10 \mathrm{ppt}$ ) among the examined alkanethiols. The relative sensitivities were 50 ppt (DSU) $<25$ ppt $\left(\mathrm{CEG}_{6}\right)<10 \mathrm{ppt}\left(\mathrm{C}_{2}-\mathrm{NTA}\right)$, which is inversely related to the tilt angle $\theta_{\mathrm{DSU}}>\theta_{\mathrm{CEG} 6}>$

$\theta_{\mathrm{C} 2-N T A}$. This suggests that the antigen with a higher tilt angle has a high affinity to Ab, but provides less sensitivity. Also, the anti-clenbuterol monoclonal antibody recognizes the tail group of clenbuterol in the molecular structure.

\section{Acknowledgements}

Suherman wishes to thank the Hitachi Scholarship Foundation for support and facilitation during the Ph.D program.

\section{Appendix A. Supplementary data}

Supplementary data associated with this article can be found, in the online version, at http: 


\section{References}

1. X. Fan, I.M. White, S.I. Shopova, H. Zhu, Sensitive optical biosensors for unlabeled targets: A review, Anal. Chim. Acta 620 (2008) 8-26.

2. S.B. VanEngelenburg, A.E. Palmer, Fluorescent biosensors of protein function, Curr. Opin. Chem. Biol. 12 (2008) 60-65.

3. A. Villaverde, Allosteric enzymes as biosensors for molecular diagnosis, FEBS Lett. 554 (2003) 167-172.

4. A.K. Trilling, J. Beekwilder, H. Zuilhof, Antibody orientation on biosensor surfaces: minireview, Analyst 138 (2013) 1619-1627.

5. A.R. Wheeler, S. Chah, R.J. Whelan, R.N. Zare, Poly(dimethylsiloxane) microfluidic flow cells for surface plasmon resonance spectroscopy, Sens. Actuators B 98 (2004) 208-214.

6. C.C.L. Loureiro, A.M.N. Lima, H. Neff, Optical monitoring of micro fluidic flow conditions, employing surface plasmon resonance sensing, J. Phys.: Conference Ser. 85 (2007) 1-8.

7. C.J. Huang, W. Knoll, A. Sessitsch, J. Dostalek, SPR bacterial pathogen biosensor: The importance of fluidic condition and probing depth, Talanta 122 (2014) 166-171.

8. W. Putzbach, N.J. Ronkainen, Immobilization technique in the fabrication of nanomaterial-based electrochemical biosensors: A review, Sensors 13 (2013) $4811-4840$. 
9. M. Joshi, N. Kale, V.R. Rao, S. Mukherji, A novel dry method for surface modification of SU-8 for immobilization of biomolecules in Bio-MEMS, Biosens. Biolelectron. 22 (2007) 2429-2435.

10. C.W. Chiu, X. He, H. Liang, Surface modification of a neural sensor using graphene, Electrochim. Acta 94 (2013) 42-48.

11. S. Yeo, C. Choi, C.W. Jang, S. Lee, Y.M. Jhon, Sensitivity enhancement of carbon nanotube based ammonium ion sensors through surface modification by using oxygen plasma treatment, Appl. Phys. Lett. 102 (2013) 073106.

12. J. Pribyl, M. Hepel, J. Halamek, P. Skladal, Development of piezoelectric immunosensors for competitive and direct determination of atrazine, Sens. Actuators B 91 (2003) 333-341.

13. M. Liu, B. Ning, L. Qu, Y. Peng, J. Dong, N. Gao, L. Liu, Z. Ghao, Development of indirect competitive immunoassay for highly sensitive determination of ractopamine in pork liver samples based on surface plasmon resonance sensor, Sens. Actuators B 161 (2012) 124-130.

14. D.R. Shankaran, K.V. Gobi, N. Miura, Recent advancements in surface plasmon resonance immunosensors for detection of small molecules of biomedical, food and environmental interest, Sens. Actuators B 121 (2007) 158-177.

15. X.D. Hoa, A.G. Kirk, M. Tabrizian, Towards integrated and sensitive surface plasmon resonance biosensors: A review of recent progress, Biosens. Biolelectron. 23 (2007) 151-160.

16. M.G. Manera, E.F. Vila, J.M.G. Martin, A.G. Martin, R. Rella, Enhanced antibody recognition with a magneto-optic surface plasmon resonance (MO-SPR) sensor, Biosens. Biolelectron. 58 (2014) 114-120. 
17. P. Koegler, A. Clayton, H. Thissen, G.N.C. Santos, P. Kingshott, The influence of nanostructured materials on biointerfacial interactions, Adv. Drug Delivery Rev. 64 (2012) 1820-1839.

18. N.K. Chaki, K. Vijayamohanan, Self-assembled monolayers as a tunable platform for biosensor applications, Biosens. Biolelectron. 17 (2002) 1-12.

19. S.K. Arya, P.R. Solanki, M. Datta, B.D. Malhotra, Recent advances in selfassembled monolayers based biomolecular electronic devices, Biosens. Biolelectron. 24 (2009) 2810-2817

20. V. Chaudhari, H.M.N. Kotresh, S. Srinivasan, V.A. Esaulov, Substitutional selfassembly of alkanethiol and selenol SAMs from a lying-down doubly tethered butanedithiol SAM on gold, J. Phys. Chem. C 115 (2011) 16518-16523.

21. S. Suherman, K. Morita, T. Kawaguchi, Surface plasmon resonance for detecting clenbuterol: Influence of monolayer stucture, Unpublished results.

22. S. Chen, L. Li, C.L. Boozer, S. Jiang, Controlled chemical and structural properties of mixed self-assembled monolayers of alkanethiols on Au(111), Langmuir 16 (2000) 9287-9293.

23. A.V. Shevade, J. Zhou, M.T. Zin, S. Jiang, Phase behavior of self-assembled monolayers of alkanethiols on $\mathrm{Au}(111)$ : A configuration-bias Monte Carlo simulation study, Langmuir 17 (2001) 7566-7572.

24. N.J. Brewer, G.J. Leggett, Chemical force microscopy of mixed self-assembled monolayers of alkanethiols on gold: Evidence for phase separation, Langmuir 20 (2004) 4109-4115.

25. N.M. Grubor, R. Shinar, R. Jankowiak, M.D. Porter, G.J. Small, Novel biosensor chip for simultaneous detection of DNA-carcinogen adducts with lowtemperature fluorescence, Biosens. Biolelectron. 19 (2004) 547-556. 
26. M.D. Porter, R.J. Lipert, L.M. Siperko, G. Wang, R. Narayanan, SERS as a bioassay platform: Fundamentals, design, and applications, Chem. Soc. Rev. 37 (2008) 1001-1011.

27. J.M. Encarnacao, L. Rosa, R. Rodrigues, L. Pedro, F.A. da Silva, J. Goncalves, G.N.M. Ferreira, Piezoelectric biosensors for biorecognition analysis: Application to the kinetic study of HIV-1 Vit protein binding to recombinant antibodies, J. Biotechnol. 132 (2007) 142-148.

28. Y. Zhang, Y. Chen, G. Jin, Serum tumor marker detection on PEGlated lipid membrane using biosensor based on total internal reflection imaging ellipsometry, Sens. Actuators B 159 (2011) 121-125.

29. K. Nagatomo, T. Kawaguchi, N. Miura, K. Toko, K. Matsumoto, Development of a sensitive surface plasmon resonance immunosensor for detection of 2,4dinitrotoluene with a novel oligo (ethylene glycol)-based sensor surface, Talanta 79 (2009) 1142-1148.

30. T. Kawaguchi, D.R. Shankaran, S.J. Kim, K.V. Gobi, K. Matsumoto, K. Toko, N. Miura, Fabrication of a novel immunosensor using functionalized self-assembled monolayer for trace level detection of TNT by surface plasmon resonance, Talanta 72 (2007) 554-560.

31. X. Lou, L. He, Surface passivation using oligo(ethylene glycol) in ATRP-assisted DNA detection, Sens. Actuators B 129 (2008) 225-230.

32. S.M. Grayson, J.M.J. Frechet, Convergent dendrons and dendrimers: From synthesis to application, Chem. Rev. 101 (2001) 3819-3867.

33. C. Juan, C. Igualada, F. Moragues, N. leon, J. Manes, Development and validation of a liquid chromatography tandem mass spectrometry method for the 
analysis of ß-agonists in animal feed and drinking water, J. Chromatogr. A 1217 (2010) 6061-6068.

34. C. Li, Y-L. Wu, T. Yang, Y. Zhang, W-G. Huang-Fu, Simultaneous determination of clenbuterol, salbutamol and ractopamine in milk by reversedphase liquid chromatography tandem mass spectrometry with isotop dilution, J. Chromatogr. A 1217 (2010) 7873-7877.

35. T. Kawaguchi, D.R. Shankaran, S.J. Kim, K. Matsumoto, K. Toko, N. Miura, Surface plasmon resonance immunosensor using Au nanoparticle for detection of TNT, Sens. Actuators B 133 (2008) 467-472.

36. D.R. Shankaran, K. Matsumoto, K. Toko, N. Miura, Development and comparison of two immunoassays for the detection of 2,4,6-trinitrotoluene (TNT) based on surface plasmon resonance, Sens. Actuators B 114 (2006) 71-79.

37. K. Matsumoto, A. Torimaru, S. Ishitobi, T. Sakai, H. Ishikawa, K. Toko, N. Miura, T. Imato, Preparation and characterization of a polyclonal antibody from rabbit for detection of trinitrotoluene by a surface plasmon resonance biosensor, Talanta 68 (2005) 305-311.

38. K. Shimazu, T. Kawaguchi, T. Isomura, Construction of mixed mercaptopropionic acid/alkanethiol monolayers of controlled composition by structural control of a gold substrate with underpotentially deposited lead atoms, J. Am. Chem. Soc. 124 (2002) 652-661.

39. K. Shimazu, Y. Hashimoto, T. Kawaguchi, K. Tada, Construction of mixed mercaptopropionic acid/alkanethiol monolayers on polycrystalline gold electrodes using underpotentially deposited lead as the control element, J. Electroanal. Chem. 523 (2002) 163-169. 
40. C.J. Zhong, J. Zak, M.D. Porter, Voltammetric reductive desorption characteristics of alkanethiolate monolayers at single crystal Au(111) and (110) electrode surfaces, J. Electroanal. Chem. 421 (1997) 9-13.

41. M.M. Walczak, C.A. Alves, B.D. Lamp, M.D. Porter, Electrochemical and X-ray photoelectron spectroscopic evidence for differences in the binding sites of alkanethiolate monolayers chemisorbed at gold, J. Electroanal. Chem. 396 (1995) $103-114$.

42. Y. Li, J. Ren, H. Nakajima, B.K. Kim, N. Soh, K Nakano, T. Imato, Flow sandwich immunoassay for spesific anti-OVA IgG antibody by use of surface plasmon resonance sensor, Talanta 77 (2008) 473-478.

43. H. Aizawa, Y. Gokita, J.W. Park, Y. Yoshimi, S. Kurosawa, Antibody immobilization on functional monolayers using a quartz crystal microbalance, IEEE Sens. J. 6 (2006) 1052-1056.

44. N. Soh, T. Tokudo, T. Watanabe, K. Mishima, T. Imato, T. Masadome, Y. Asano, S. Okutani, O. Niwa, S. Brown, A surface plasmon resonance immunosensor for detecting a dioxin precursor using a gold binding polypeptide. Talanta 60 (2003) 733-745.

45. G. Sakai, S. Nakata, T. Uda, N. Miura, N. Yamazoe, Highly selective and sensitive SPR immunosensor for detection of methamphetamine. Electrochim. Acta 44 (1999) 3849-3854.

46. G. Sakai, K. Ogata, T. Uda, N. Miura, N. Yamazoe, A surface plasmon resonance-based immunosensor for highly sensitive detection of morphine. Sens. Actuators B Chem. 49 (1998) 5-12. 
Table 1. Surface concentration of various alkanethiol monolayers and the immobilized antigen calculated by different methods.

\begin{tabular}{lcccc}
\hline \multirow{2}{*}{ Thiols } & \multicolumn{4}{c}{$\begin{array}{c}\text { Surface concentration } \\
\left(\times 10^{-10} \mathrm{~mol} \mathrm{~cm}^{-2}\right)\end{array}$} \\
\cline { 2 - 5 } & Thiol & $\begin{array}{c}\text { SPR } \\
\text { Immobilized Antigen }\end{array}$ & $\begin{array}{c}\text { Electrochemistry } \\
\text { Thiol }\end{array}$ & $\begin{array}{c}\text { STM } \\
\text { Thiol }\end{array}$ \\
\cline { 2 - 5 } & Immersion & $0.71 \pm 0.16$ & $6.6 \pm 0.4$ & $5.6 \pm 0.2$ \\
$\mathrm{CEG}_{6}$ & $5.5 \pm 0.1$ & $1.01 \pm 0.07$ & $8.5 \pm 0.5$ & $6.2 \pm 0.6$ \\
$\mathrm{C}_{2}$-NTA & $2.6 \pm 0.1$ & $1.25 \pm 0.04$ & $4.6 \pm 0.2$ & $2.8 \pm 0.1$ \\
\hline
\end{tabular}



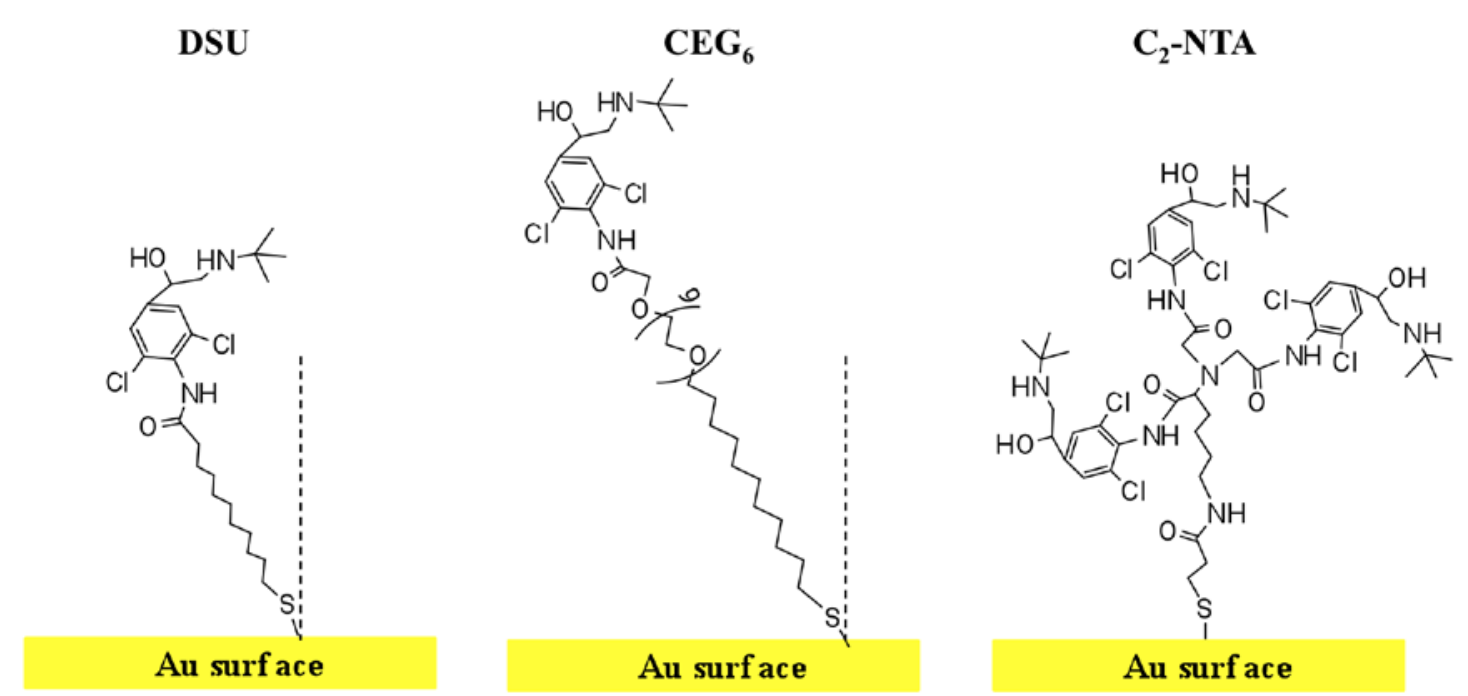

Fig. 1. Orientation of DSU, $\mathrm{CEG}_{6}$, and $\mathrm{C}_{2}$-NTA thiols on Au surface. 

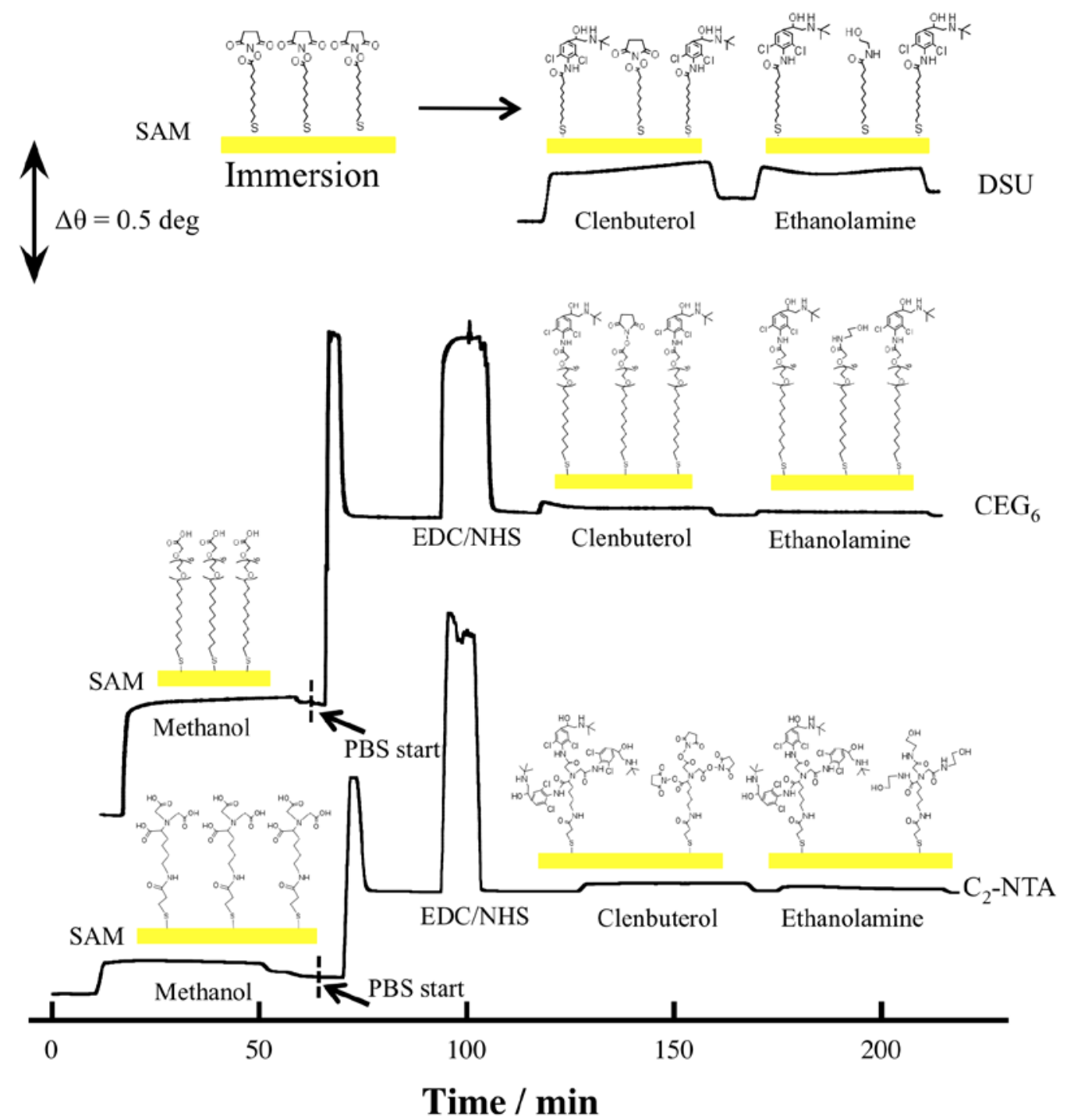

Fig. 2. SPR sensorgrams for fabrication of the sensor surface using various functionalized thiol monolayer (DSU, $\mathrm{C}_{2}-\mathrm{NTA}$ and $\mathrm{CEG}_{6}$ ) on an Au surface. Flow rate was $5 \mu \mathrm{L} \mathrm{min}^{-1}$. 


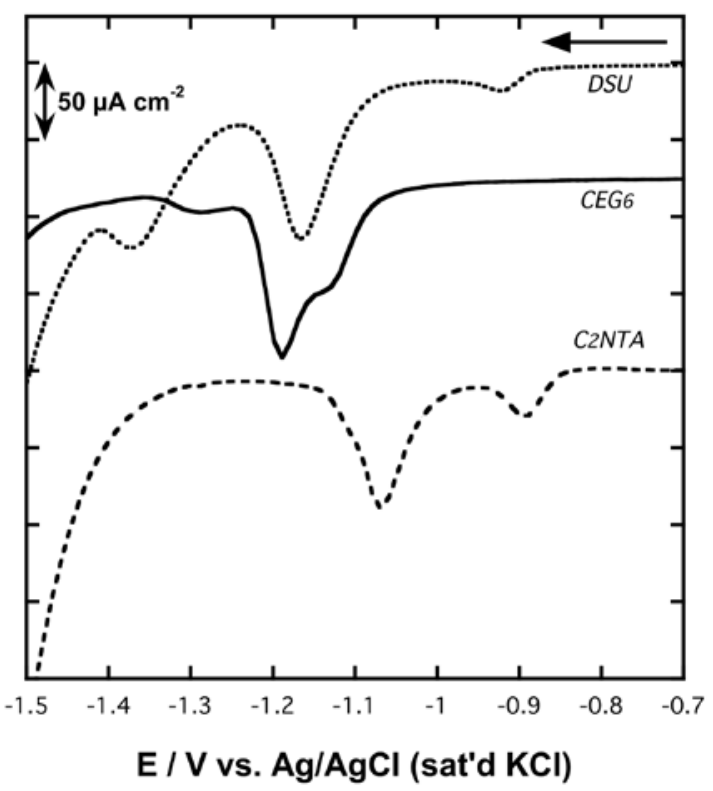

Fig. 3. Voltammograms for reductive desorption of DSU, CEG 6 and $\mathrm{C}_{2}-\mathrm{NTA}$ monolayers. Electrolyte solution was $0.1 \mathrm{M} \mathrm{KOH}$, while sweep rate was $0.1 \mathrm{~V} \mathrm{~s}^{-1}$. 

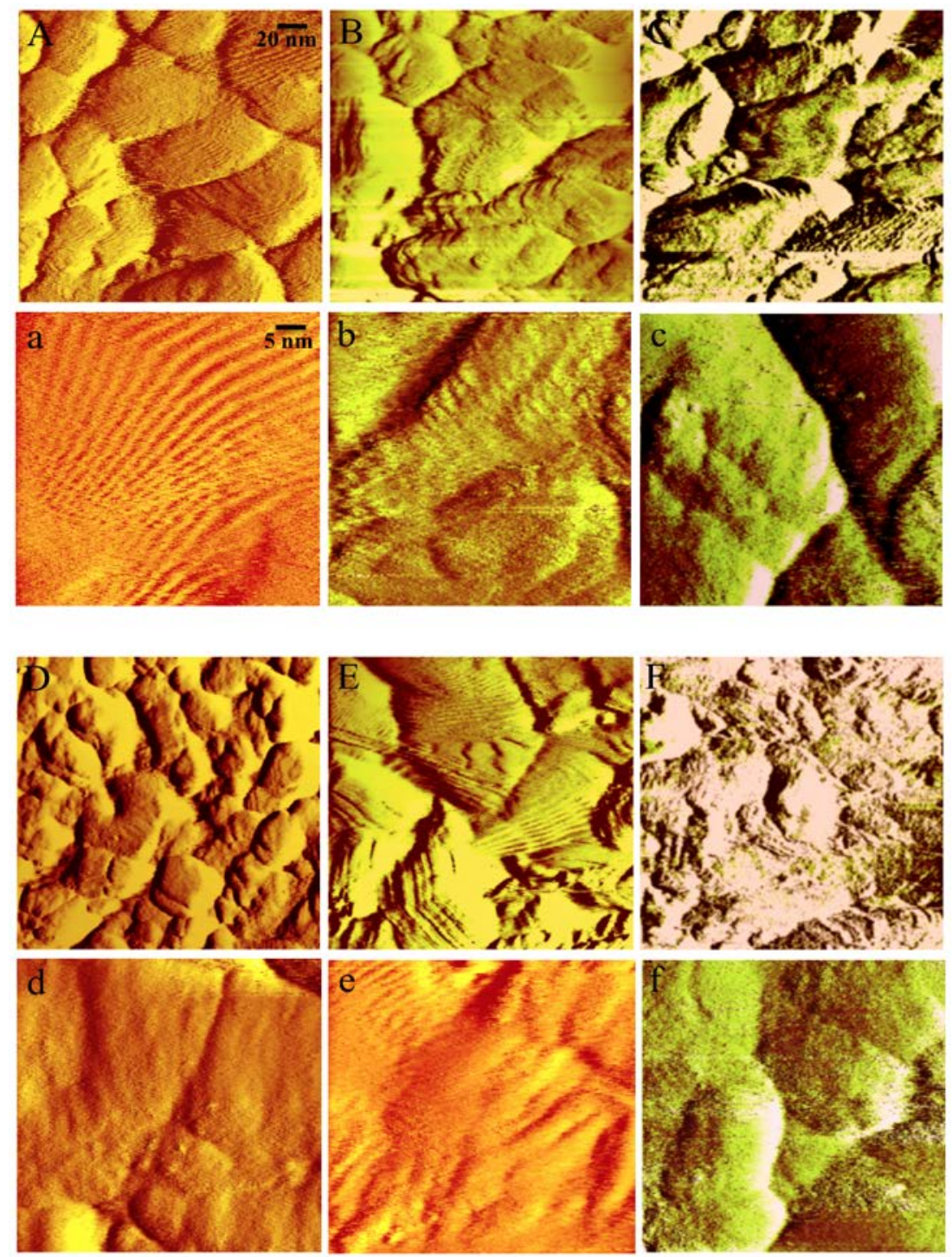

Fig. 4. High-resolution STM images of DSU monolayer (A, a), $\mathrm{CEG}_{6}$ monolayer (B, b) and $\mathrm{C}_{2}$-NTA monolayer $(\mathrm{C}, \mathrm{c})$; more over DSU sensor surface $(\mathrm{D}, \mathrm{d})$, ), $\mathrm{CEG}_{6}$ sensor surface $(\mathrm{E}, \mathrm{e})$ and $\mathrm{C}_{2}$-NTA sensor surface $(\mathrm{F}, \mathrm{f})$ on polycrystalline Au. The scales for the top and bottom images for each group are $200 \mathrm{~nm}$ x $200 \mathrm{~nm}$ and $50 \mathrm{~nm}$ $\mathrm{x} 50 \mathrm{~nm}$, respectively. 

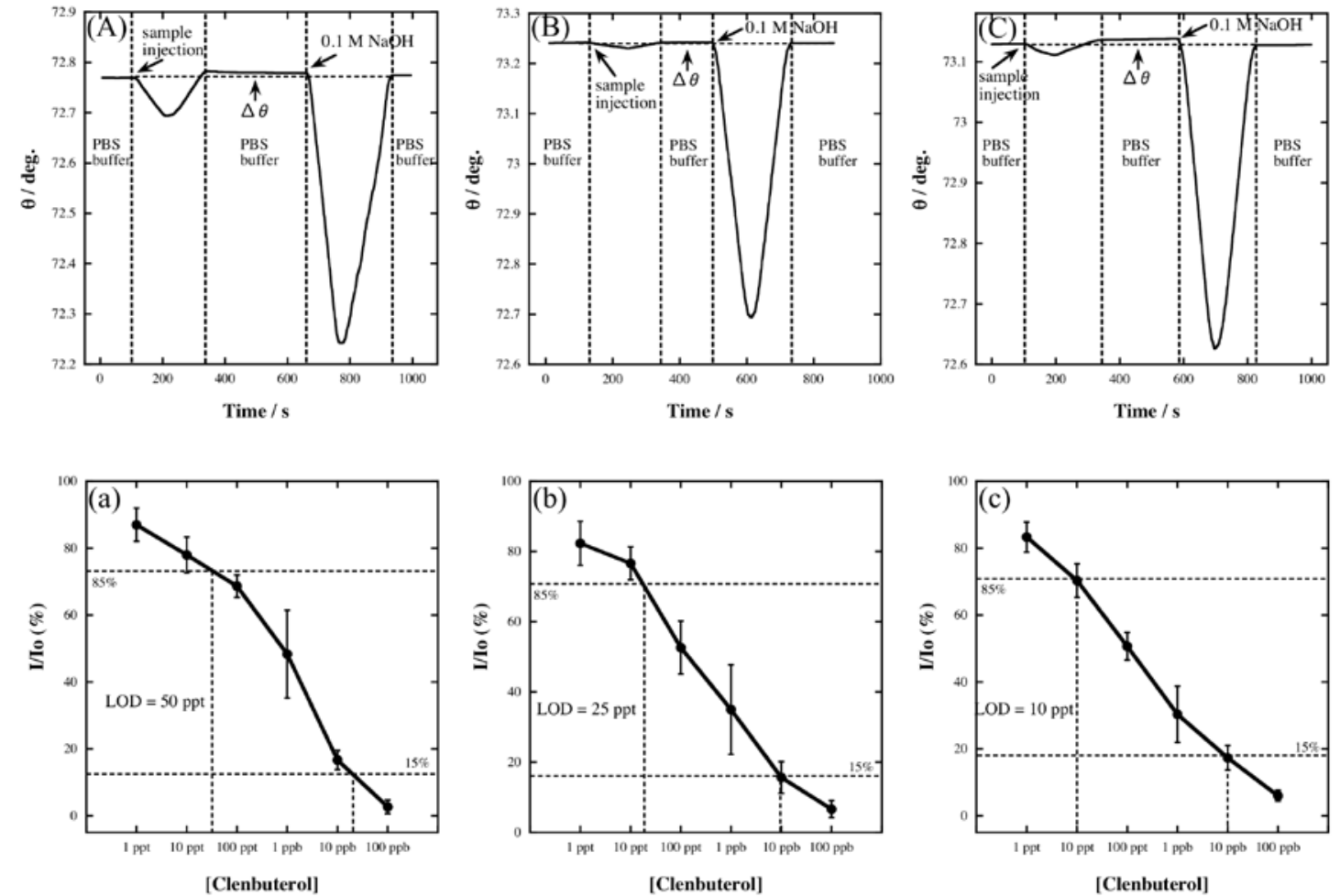

Fig. 5. SPR sensing format for immunoreaction at (A) DSU, (B) $\mathrm{CEG}_{6}$ and (C) $\mathrm{C}_{2}-$ NTA sensor surfaces. Clenbuterol detection by using indirect competitive inhibition immunoassay on sensor surfaces at the flow rate of $100 \mu \mathrm{L} \mathrm{min}^{-1}$ : (a) DSU, (b) $\mathrm{CEG}_{6}$ and (c) $\mathrm{C}_{2}$ NTA. Inhibition rate was calculated from the angle shift in the presence (I)/absence $\left(\mathrm{I}_{0}\right)$ of clenbuterol. In the premixing process, 1 ppm Ab was mixed with series of clenbuterol concentrations. The incubation time was 2 min. 

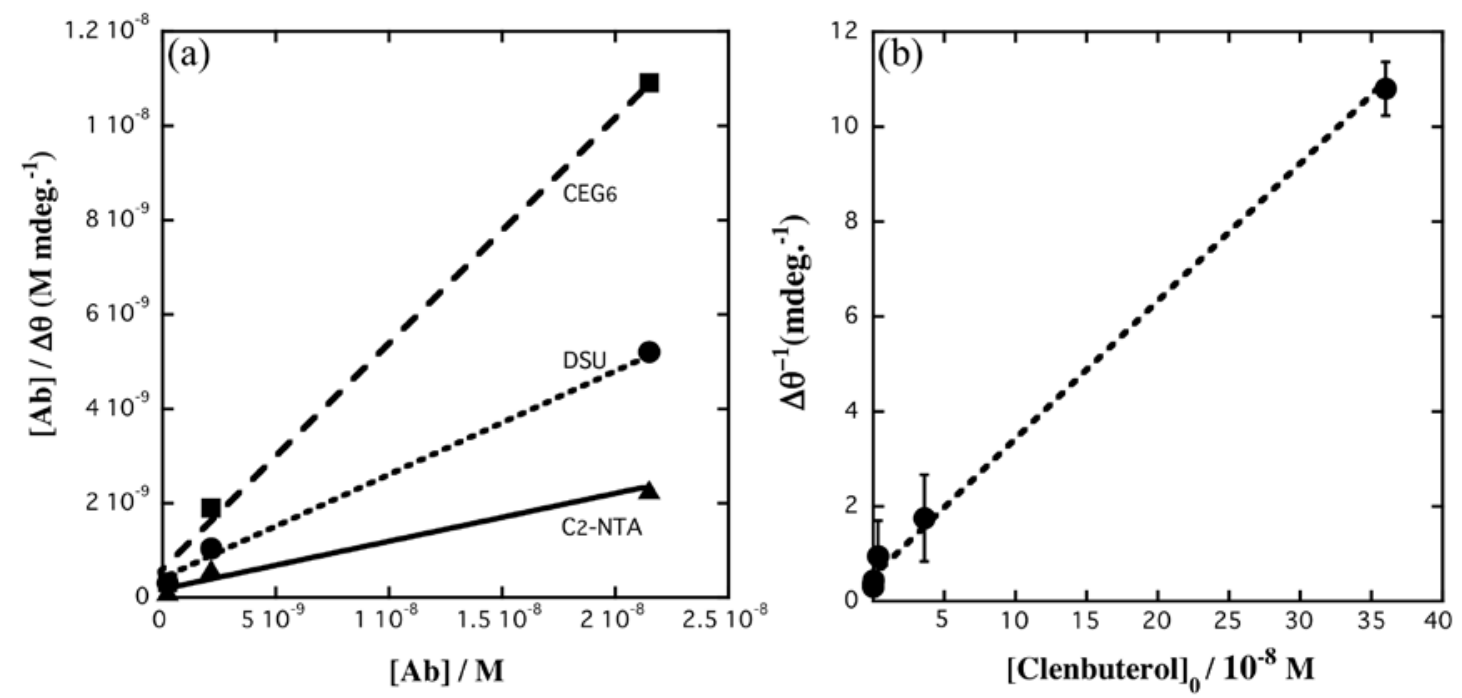

Fig. 6. (a) Langmuir adsorption isotherm plot for binding of anti-clenbuterol antibody (Ab) to clenbuterol immobilized on the sensor surface, and (b) clenbuterol solution concentration dependence using indirect competitive inhibition immunoassay. 